\title{
DOCUMENTO DE VOLUNTADES ANTICIPADAS
}

\author{
Grup D’Opinió de l'Observatori de Bioètica i Dret,
}

Universitat de Barcelona, España

\section{Presentación}

El Grupo de Opinión del Observatori de Bioètica i Dret, con sede en el Parc Científic de Barcelona, surgió para tratar de dar respuesta a la necesidad de analizar científicamente y desde un punto de vista pluridisciplinar, las implicaciones éticas, sociales y jurídicas de las biotecnologías, y para proponer después actuaciones concretas.

La creación del Observatori de Bioètica i Dret se llevó a cabo con la intención de participar en el diálogo universidad-sociedad, con la finalidad de hacer oír su voz en el conjunto de la comunidad y, más específicamente, ante los organismos públicos, administrativos o políticos que regulan y controlan las actividades de investigación y la aplicación de las nuevas tecnologías. Ello requiere también la voluntad de establecer relación con los medios de comunicación, para mejorar la calidad de la información. Para ello se han elaborado ya diversos Documentos sobre temas de actualidad en los que no existía una opinión unánime en la sociedad, ni tampoco en las diversas comunidades científicas implicadas.

En esta ocasión el grupo presenta un Documento que pretende aportar pautas útiles a los ciudadanos, a los centros asistenciales y a la administración, al proporcionar un modelo de Documento de Voluntades Anticipadas, en el que cada uno pueda acomodar y redactar las propias previsiones respecto a las actuaciones sanitarias que desee recibir, si se presentan determinadas circunstancias y se carece de conciencia para manifestar personalmente la propia voluntad; con él se aspira a facilitar la tarea de quienes decidan hacer uso de las nuevas posibilidades de ejercicio de la autonomía que la ley reconoce.

Para la elaboración de esta propuesta, el grupo ha sido coordinado por el Dr. Albert Royes i Qui, Profesor de Ética Médica de la Facultad de Medicina de la UB, y ha contado con la aportación de los especialistas en Medicina, en Enfermería, en Filosofía, en Biología, en Antropología y en Derecho, que se relacionan al final de este Documento.

\section{Preámbulo}

El respeto a la libertad de la persona y a los derechos de los pacientes adquiere una especial relevancia en el marco de las relaciones asistenciales, ámbito en el que la autonomía de la persona constituye un elemento central cuyas manifestaciones más evidentes, pero no exclusivas, se plasman en la necesidad de suministrar información y de recabar el consentimiento de las personas enfermas. Una de las repercusiones de mayor interés consiste en la posibilidad de establecer previsiones sobre las actuaciones terapéuticas que se desee recibir -o no- en el futuro, posibilidad que ahora se abre a los ciudadanos como consecuencia de las últimas decisiones normativas habidas en nuestro país.

Así, el día 11 de enero de 2001 el Diario Oficial de la Generalitat de Catalunya publicaba la Ley 21/2000, de 29 de diciembre, "sobre los derechos de información concernientes a la salud y la autonomía del paciente, y la documentación clínica", en cuyo Art. 8 se regulan las voluntades anticipadas, siguiendo lo establecido en el Convenio sobre Derechos Humanos y Biomedicina -en vigor en nuestro país desde el 1 de enero de 
2000-, en cuyo Art. 9 se instituye que «Se tendrán en consideración los deseos expresados con anterioridad respecto a una intervención médica por un paciente que, en el momento de la intervención, no esté en condiciones de hacer saber su voluntad". En esta misma línea, otras Comunidades Autónomas están elaborando disposiciones análogas, e incluso se ha presentado ante el Senado una Proposición de Ley de contenido semejante que cuenta con el respaldo de todos los grupos parlamentarios.

La posibilidad de otorgar un Documento de voluntades anticipadas, junto con la exigencia del consentimiento informado, modificará profundamente la tradición en las relaciones sanitarias dentro de nuestra cultura, dominada por el paternalismo médico, actitud que privaba a las personas de algunos de sus derechos, en cuanto se convertían en pacientes. Es preciso valorar positivamente esta voluntad de cambio en las relaciones asistenciales, si bien hay que tener en cuenta que una innovación de tal envergadura -en hábitos tan arraigados- no es de esperar que tenga lugar rápidamente y requerirá del empeño educativo de los responsables de las instituciones sanitarias, en especial de los Comités de Ética Asistencial, así como de una decidida actitud de los ciudadanos en el ejercicio del derecho a establecer sus voluntades.

Por otra parte, no conviene ignorar las dificultades añadidas de carácter práctico que existen para que los documentos de voluntades anticipadas sean accesibles en los momentos y lugares en que se requiera conocer su existencia y su contenido. Por ello, aunque la Ley determina que se hagan llegar al centro sanitario en que la persona sea atendida y que se incorporen a la historia clínica, para que la previsión establecida sea verdaderamente efectiva conviene contar con un Registro de Documentos fácilmente accesible a los profesionales sanitarios autorizados, lo cual resulta especialmente necesario en los casos de urgencia o accidente.
Con el Documento de Voluntades Anticipadas que se presenta se pretende ofrecer a quien desee hacer uso de este derecho- un modelo que contenga los elementos formales y materiales previstos por la Ley.

\section{Declaración}

\section{Exposición de motivos}

Considerando que la regulación de los Documentos de Voluntades Anticipadas representa un importante paso adelante en el respeto a la autonomía de las personas -al permitirles decidir respecto a las actuaciones sanitarias que deseen recibir, o no, en el futuro si se encuentran en unas determinadas circunstancias-, ya que la ley reconoce que constituyen «un elemento coadyuvante en la toma de decisiones, a fin de conocer con más exactitud la voluntad del paciente» cuando éste no se encuentre en condiciones de expresarla directamente en el momento de necesitar una actuación sanitaria.

Considerando que con el reconocimiento legal de esta posibilidad nuestro país se une a la lista de sociedades en que los ciudadanos pueden hacer uso de una opción, largo tiempo deseada, que se incardina en el proceso de concreción de los Derechos $\mathrm{Hu}$ manos en la sanidad, ámbito en el que la autonomía de las personas es un valor de primer orden.

Considerando que los profesionales sanitarios y los centros asistenciales deberán habituarse a la existencia, cada vez más frecuente, de personas que hayan otorgado un Documento de Voluntades Anticipadas, lo que requerirá tratar abiertamente sobre tal posibilidad -en especial en casos de pacientes con enfermedades crónicas o degenerativas- y conocer el contenido del Documento, a fin de respetar la voluntad de los pacientes cuando estos no puedan manifestarla de manera inmediata.

Considerando que hay aspectos de la Ley que resultan equívocos e inclusive contra- 
dictorios con el espíritu de la misma, ya que, a tenor de lo que se expone en su Preámbulo, conviene resaltar la dificultad de comprender-en este contexto-manifestaciones como las que se recogen en el apartado 3 del Artículo 8 según el cual "no se pueden tener en cuenta voluntades anticipadas que incorporen previsiones contrarias al ordenamiento jurídico o a la buena práctica clínica, o que no se correspondan exactamente con el supuesto del hecho que el sujeto ha previsto a la hora de emitirlas".

Porque, en primer lugar, es obvio que el marco legal de cualquier sociedad puede variar durante el intervalo de tiempo que transcurra entre el momento de otorgar un Documento de voluntades anticipadas y el de hacer efectivo su contenido; consecuentemente, serían aceptables previsiones que, aun no estando de acuerdo actualmente con el ordenamiento jurídico vigente, en un futuro puedan tener cabida dentro de la legalidad.

Porque, en segundo lugar, la mención a conceptos abiertos -como que no se tendrán en cuenta voluntades contrarias "a la buena práctica clínica" sin que se especifique en ninguna parte cómo y quién define, en cada momento, qué se ha de entender por buena práctica clínica- puede dejar sin efecto la voluntad expresada por el otorgante del Documento si el personal sanitario o las asociaciones profesionales entienden que tienen la facultad de interpretar la adecuación del contenido del Documento de Voluntades Anticipadas a una imprecisa "buena práctica clínica". Es decir, vuelve a aparecer -y ahora en un texto legal- la actitud injustificadamente paternalista que una norma dirigida precisamente a preservar la autonomía de los pacientes habría tenido que abandonar sin ambigüedad.

\section{Conclusiones}

Considerando que, para que todo esto sea efectivo, es necesario concretar las posibilidades que la Ley otorga mediante algún mo- delo de documento y, además, cumplir las formalidades para legalizarlo.

El Grupo de Opinión del Observatori de Bioètica i Dret ha llegado a las siguientes conclusiones:

1. Es necesario fomentar la adopción de estas decisiones previas facilitando su otorgamiento a personas y centros, para lo cual es útil que existan modelos de Documentos de Voluntades Anticipadas a la disposición de quienes deseen acogerse al derecho de otorgarlo, y que, respetando siempre la multiplicidad de contenidos posibles, sirvan de esquema básico en el que inspirarse para la elaboración de un documento propio o bien para modificarlo a voluntad.

Para ello, este Grupo de Opinión presenta en el ANEXO un Documento de Voluntades Anticipadas estructurado en dos partes:

- la primera es una Hoja de instrucciones en la que se suministran orientaciones para las personas interesadas en otorgar un Documento de Voluntades Anticipadas, $\mathrm{y}$

- la segunda es el Documento propiamente dicho, que será necesario rellenar, firmar y formalizar como el otorgante crea más conveniente y de acuerdo con alguno de los procedimientos que establece la Ley.

2. Es conveniente hacer especial hincapié en la designación del representante como garante e intérprete del cumplimiento de las voluntades expresadas en el Documento de Voluntades Anticipadas.

El representante, como persona de confianza del otorgante, que conoce su jerarquía de valores y sus opciones vitales, será quien mejor podrá conocer y manifestar su voluntad cuando éste no pueda hacerlo personalmente.

3. Se debe promover y regular el establecimiento de un Registro General de Documentos de Voluntades Anticipadas.

Este Documento debe ser fácilmente ac- 
cesible para quienes tengan que prestar atención sanitaria en un momento dado al otorgante y para los con él vinculados.

4. Se recomienda que el documento sea otorgado ante notario con la finalidad de dotarlo de un mayor grado de vinculatoriedad.

Así, al menos mientras no se creen y entren en funcionamiento efectivo los Registros que se proponen en la anterior conclusión, estimamos que la manifestación de voluntad realizada ante fedatario público debe tener mayor fuerza persuasiva a la hora de asegurar que será respetada por médicos y familiares, ya que la presencia del notario garantiza legalmente la capacidad del sujeto en el momento de suscribirlo.

\section{ANEXO}

Instrucciones para rellenar el Documento de Voluntades Anticipadas:

\section{Respecto a los objetivos del Documento}

Este Documento quiere dejar constancia fehaciente, y por escrito, de su voluntad, para cuando usted no pueda manifestarla directamente, en relación con las actuaciones sanitarias que desearía recibir, o no recibir, en determinados supuestos.

\section{Respecto al procedimiento a seguir}

Es muy recomendable que lea cuidadosamente el contenido y que, si cree que puede serle de ayuda, pida consejo con el fin de concretarlo a su caso personal.

Si es su deseo, puede modificar cualquier aspecto de su contenido, o bien añadir cualquier consideración que crea oportuna. Con este fin, se han dejado espacios en blanco en el apartado de Instrucciones adicionales.

Ha de saber que este Documento puede ser invalidado en cualquier momento, pero solamente por usted. Puede hacerlo bien sea al final del Documento, donde hay un espacio previsto para formalizar por escrito la revocación, o bien en cualquier otro documento firmado por usted. Conviene que su voluntad conste siempre de manera fehaciente para cuando no pueda expresarla por sí mismo, y recuerde que si usted está en uso de sus facultades ya no será necesario acudir a lo estipulado en sus voluntades anticipadas, pues podrá manifestarlas directamente.

Tenga en cuenta que es extremadamente importante la designación de un representante -y un substituto para el caso de que aquél se hallase imposibilitado para ejercer su función por cualquier causa- ya que será la persona designada para resolver cualquier incertidumbre en la interpretación de su voluntad. Es preciso que sean personas de su máxima confianza, pero no es necesario que formen parte de su familia. Estas personas deben conocer, con la máxima precisión, su intención al otorgar este Documento y cuáles pueden llegar a ser sus funciones.

\section{Respecto al contenido del Documento}

En el Documento que presentamos se han previsto algunos supuestos típicos. Pero dependiendo de su situación de salud, actual o futura, usted mismo puede considerar la conveniencia de añadir otras posibilidades que puedan precisar mejor sus previsiones.

En este Documento se hace referencia a la voluntad que "no me sean aplicadas $-o$ bien que se retiren si ya se han instaurado medidas de soporte vital'. Bajo tal denominación se incluyen habitualmente las siguientes: reanimación cardio-respiratoria, conexión a un respirador mecánico, nutrición e hidratación artificiales, o cualquier otra que, si no se instauran o si se retiran, la vida se compromete a muy corto plazo.

Además, usted puede hacer constar, en el apartado de Instrucciones adicionales, 
otras voluntades, como por ejemplo si no desea ser trasladado a un hospital y prefiere ser atendido en su domicilio, si quiere recibir asistencia religiosa, si hay que advertir de su situación a algunas personas concretas, etc.

\section{Respecto a la tramitación del Documento}

La normativa prevé dos caminos alternativos para validar este Documento:

- Formalizarlo ante notario, como cualquier testamento; es el procedimiento que mejor puede garantizar que su voluntad será respetada.

- Firmarlo delante de tres testigos mayores de edad y con plena capacidad de obrar, teniendo en cuenta, además, que dos de ellos, como mínimo, no han de tener relación de parentesco - hasta el segundo grado-, ni tampoco vínculos patrimoniales con el otorgante. Si se hace uso de esta posibilidad, es necesario que los testigos firmen en el espacio reservado para ello en el propio Documento.

\section{Consideraciones finales}

Tenga en cuenta que su médico, sus familiares, su representante y representante sustituto han de conocer la existencia de este Documento, así como su contenido. Es muy recomendable, por tanto, que tengan copia y también que se adjunte a su historia clínica, allí donde la tenga abierta o puedan abrirla en el futuro.

No olvide poner la fecha y firmar el Documento, y hacer que tanto su representante como su representante sustituto también lo firmen, así como los testigos, si usted ha escogido esta opción para formalizarlo. 


\section{DOCUMENTO DE VOLUNTADES ANTICIPADAS}

Yo mayor de edad, con DNI núm. y domicilio en calle , con plena capacidad de obrar y actuando libremente, otorgo el presente Documento de voluntades anticipadas.

En lo que se refiere a la interpretación y aplicación de este Documento, designo mi representante a con DNI núm.

con domicilio en calle y teléfono quien deberá ser considerado como interlocutor válido y necesario para el médico o el equipo sanitario que me atienda y como garante del cumplimiento de mi voluntad aquí expresada. De la misma manera, por si se diera el caso de renuncia o imposibilidad de mi representante designo como representante sustituto a micilio en calle , con DNI núm. , y do-

\section{DISPONGO:}

Si en un futuro me encontrase en alguna de las situaciones que se señalan a continuación:

1. Demencia severa debida a cualquier causa (por ejemplo: enfermedad de Alzheimer...)

2. Daños encefálicos severos debidos a cualquier causa (por ejemplo: coma irreversible, estado vegetativo persistente,...)

3. Enfermedad degenerativa neuromuscular en fase avanzada (por ejemplo: esclerosis múltiple,...)

4. Cáncer diseminado en fase avanzada (por ejemplo: tumor maligno con metástasis,...)

5. Enfermedad inmunodeficiente en fase avanzada (por ejemplo: SIDA,...)

6. (otras)

Y si a juicio de los médicos que entonces me atiendan (siendo por lo menos uno de ellos especialista) no hay expectativas de recuperación sin que se sigan secuelas que impidan una vida digna según yo la entiendo, mi voluntad es que NO sean aplicadas -o bien que se retiren si ya han empezado a aplicarse-medidas de soporte vital o cualquiera otras que intenten prolongar mi supervivencia.

DESEO, en cambio, que se instauren las medidas que sean necesarias para el control de cualquier síntoma que pueda ser causa de dolor, padecimiento o malestar, aunque eso pueda:

a) Acortar mi expectativa de vida.

b) Poner fin a mi vida.

Instrucciones adicionales:

a)

b) 
En caso que el o los profesionales sanitarios que me atiendan aleguen motivos de conciencia para no actuar de acuerdo con mi voluntad aquí expresada, solicito ser transferido a otro u otros profesionales que estén dispuestos a respetarla.

Lugar y fecha:

Firma del otorgante:

Firma del representante:

Firma del representante sustituto:

En caso que el presente Documento se otorgue ante testigos y no ante notario:

Primer testigo: , DNI núm.

Firma:

Segundo testigo: , DNI núm.:

Firma:

Tercer testigo: , DNI núm.:

Firma:

$\mathrm{Si}$, más adelante, el otorgante quisiera dejar sin efecto el presente Documento podrá firmar, si lo desea, la siguiente orden de anulación:

Yo, , mayor de edad, con DNI núm. el presente Documento de voluntades anticipadas en todos sus extremos.

Lugar y fecha:

Firma: 


\section{RELACIÓN DE MIEMBROS DEL GRUP D’OPINIÓ DE L'OBSERVATORI DE BIOÈTICA I DRET QUE HAN ELABORADO ESTE DOCUMENTO}

María Casado. Directora de l'Observatori de Bioètica i Dret i del Màster de Bioètica $i$ Dret de la Universitat de Barcelona.

Pilar Antón. Professora Titular d'Ėtica i Legislació de l'Escola Universitària d'Infermeria. $U B$.

Montserrat Boada. Biòloga. Institut Universitari Dexeus de Barcelona.

Lídia Buisan. Metgessa. Consorci Hospitalari de la Creu Roja de l'Hospitalet.

Montserrat Busquets. Professora Titular d'Ètica i Legislació de l'Escola Universitària d'Infermeria. $U B$.

Maria Jesús Buxó. Catedràtica d'Antropologia Cultural de la UB.

Lluís Cabré. Metge. Hospital de Barcelona.

Mirentxu Corcoy. Catedràtica de Dret Penal de la Universitat Pública de Navarra.

Josep Egozcue. Catedràtic de Biologia Cel-lular de la Universitat Autònoma de Barcelona. Assessor del Consell d'Europa i de la Comissió Europea.

Maria Lourdes Frías. Notària de l'Il-lustre Col-legi de Notaris de Catalunya.

Roser Gonzàlez-Duarte. Catedràtica de Genètica de la UB.

Joaquim Martínez Montauti. Coordinador del Servei de Medicina Interna de l'Hospital de Barcelona.

M. Jesús Montoro. Catedràtica de Dret Administratiu de la UB.

Josep-Enric Rebés. President de la Comissió Jurídica Assessora de la Generalitat de Catalunya.

Encarna Roca. Catedràtica de Dret Civil de la UB.

Albert Royes. Professor d'Ètica Mèdica de la Facultat de Medicina de la UB.

Ramon Valls. Catedràtic d'Història de la Filosofia i Síndic de Greuges de la UB. 